\title{
Bilateral thalamic stroke after tonsillectomy in a patient with collateral extracranial anastomosis - case report
}

\author{
Małgorzata Milnerowicz ${ }^{A, B, D, E, F}$, Jerzy Garcarek ${ }^{A, B, D, E}$, Joanna Bladowska ${ }^{A, B, D, E}$, Marcin Mis ${ }^{A, D, E}$, \\ Aleksandra Milnerowicz ${ }^{A, D}$, Marek Sąsiadek ${ }^{A, B, D, E}$ \\ Department of General and Interventional Radiology and Neuroradiology, Wroclaw Medical University, Wroclaw, Poland
}

\begin{abstract}
Haemorrhage remains the most frequent and serious complication of tonsillectomy. When bleeding is recurrent, gushing, and ceases spontaneously, pseudoaneurysm of the injured artery in the proximity of the tonsillar bed should be suspected. Haemorrhage related to pseudoaneurysm occurs most commonly in the first 30 days after surgery. It can sometimes be excessive and requires a revision procedure such as external carotid artery (ECA) ligation or embolisation. During those procedures, ECA should be checked for possible anastomoses, otherwise the bleeding may persist despite the intervention.

We report an unusual case of a patient with recurrent post-tonsillectomy haemorrhage due to pseudoaneurysm of the facial artery, which persisted after ECA ligation because of the presence of collateral occipital-vertebral anastomosis. Due to the recurrence of bleeding episodes, endovascular treatment was implemented. However, the embolisation was complicated by bilateral thalamic stroke with unclear mechanism.

This case highlights the importance of anastomosis between ECA and the vertebrobasilar system, both in recurrence of significant post-tonsillectomy bleeding and in potential thromboembolic complications. Therefore, ECA ligation should always be accompanied by exclusion of possible anastomoses. In cases of non-life-threatening bleeding, embolisation seems to be the proper and more selective therapy.

Key words: tonsillectomy, ligation, therapeutic embolisation, postoperative haemorrhage.
\end{abstract}

\section{Introduction}

Postoperative hemorrhage after tonsillectomy is the most common and potentially life-threatening complication [1-3]. Primary hemorrhage occurs within 24 hours postoperatively and is related to surgical technique and/or coagulopathy $[2,3]$. Secondary hemorrhage occurs after 24 hours and is related to separation of slough from granulating fossa $[4,5]$. Multiple factors increase the risk of secondary hemorrhage, including older age, chronic tonsillitis and elevated postoperative mean arterial pressure [1,3]. Recurrent delayed bleeding episodes may indicate pseudoaneurysm of the injured artery in the tonsillar bed $[2,5,6]$.
According to many authors $[1,2,4,5]$ embolization of the damaged vessel seems to be a better treatment than ligation of the external carotid artery (ECA) because it combines diagnostic evaluation with direct selective therapy. However, life-threatening bleeding requires immediate ligature with exclusion of possible vascular anastomoses. Otherwise the bleeding may not cease after ECA ligation.

We present a case of a patient with delayed recurrent post-tonsillectomy hemorrhage resulting from a pseudoaneurysm of the facial artery which persisted after ECA ligation because of the collateral occipital-vertebral anastomosis and was complicated by bilateral thalamic stroke.

Correspondence address:

Dr. Małgorzata Milnerowicz, Department of General and Interventional Radiology and Neuroradiology, Wroclaw University Clinical Hospital,

213 Borowska Street, 50-556, Wroclaw, Poland, e-mail: gosia.milnerowicz@gmail.com

Authors' contribution:

A Study design · B Data collection · C Statistical analysis · D Data interpretation · E Manuscript preparation · F Literature search · G Funds collection 


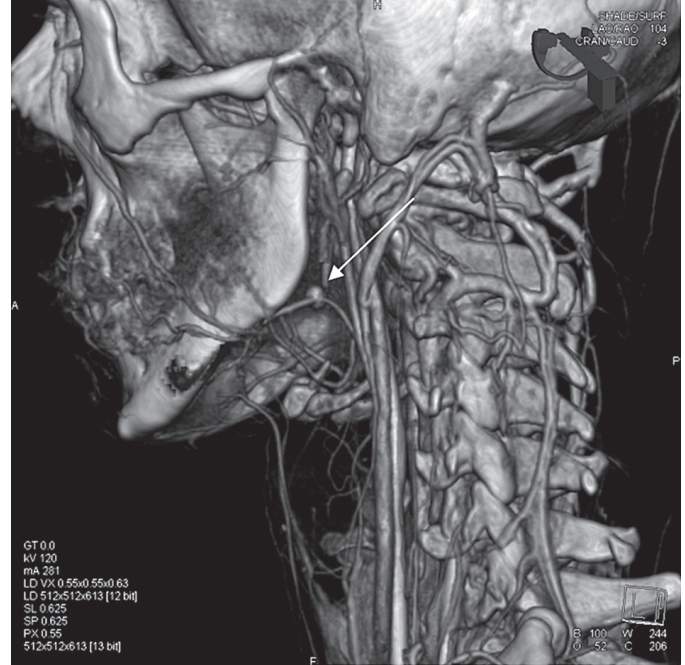

Figure 1. Computed tomography angiography of extracranial arteries. The image shows a 6-mm pseudoaneurysm of the left facial artery (arrow)

\section{Case report}

A 20-year-old patient with no significant medical history underwent adenotonsillectomy for chronic adenotonsillitis in another hospital. The procedure and imme-
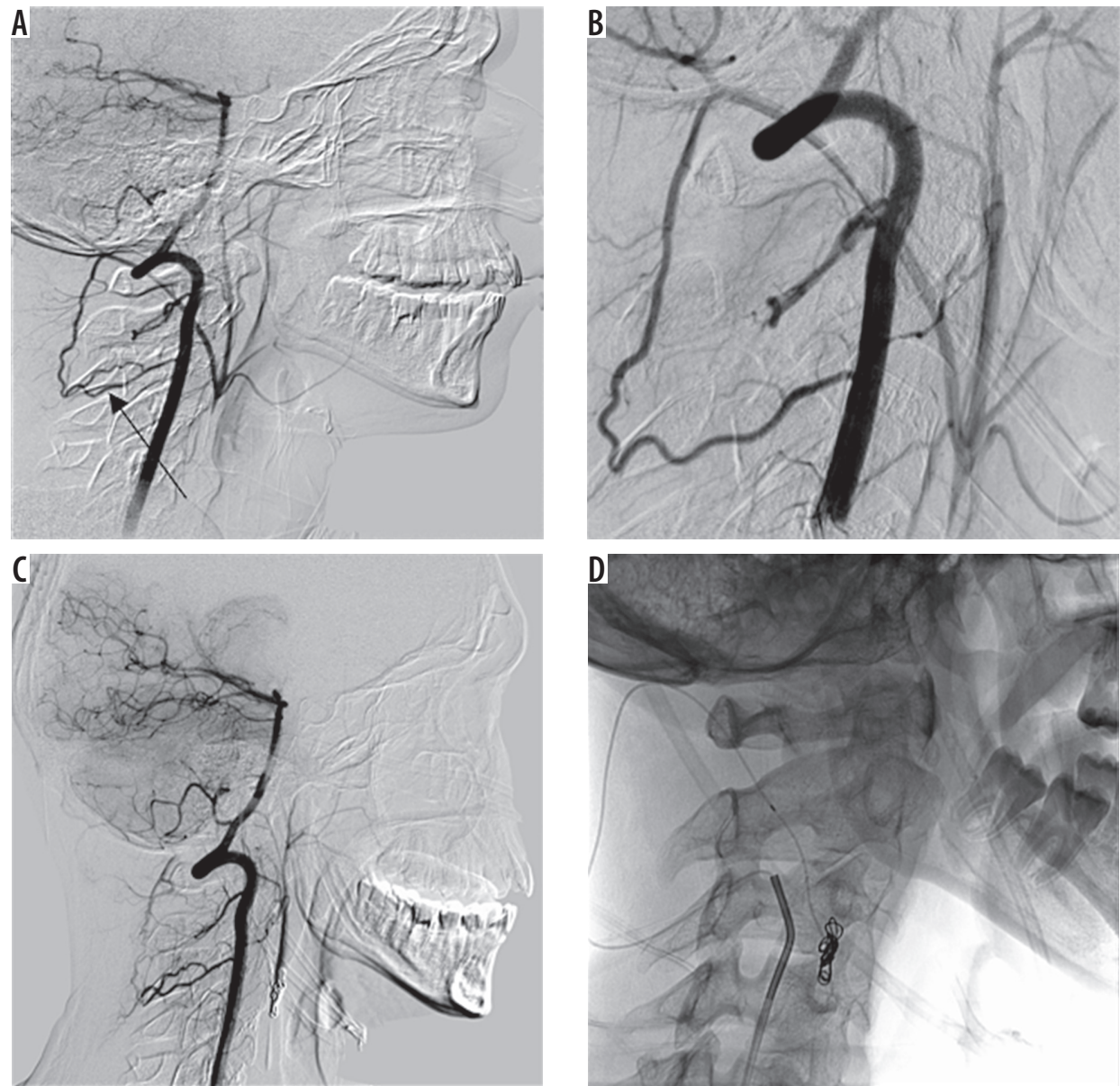

Figure 2. Digital subtraction angiography. A-B) The left vertebral artery (VA) selective angiography showed the presence of an occipital-vertebral anastomosis (arrow) between the muscular branch of the left VA and the left occipital artery with retrograde flow from the VA to the external carotid artery (ECA) in the segment above the ligation. C-D) Embolization of the trunk of the ECA was achieved by implantation of one platinum detachable coil (target $6 \mathrm{~mm} \times 15 \mathrm{~cm}$ ) and further injection of $\mathrm{N}$-butyl-2-cyanoacrylate. The final angiogram (C) demonstrated subtotal occlusion of the embolized vessel with a negligible inflow by submillimeter muscular branches 
The trunk of the ECA was selectively catheterized with a Prowler 10 microcatheter (Codman, Raynham, U.S.) via the anastomosis and subsequent embolization with a platinum detachable coil (Target, Stryker, Fremont, U.S.) was accomplished (Figure 2C). To achieve more complete embolization, N-butyl-2-cyanoacrylate (NBCA) was injected via a Magic 1.2F microcatheter (Balt, Montmorency, France). The final angiogram demonstrated subtotal occlusion of the embolized ECA trunk (Figure 2D). The procedure and immediate postoperative course were uncomplicated.

The next day the patient became lethargic with cognitive memory disorders and developed left-sided hemiparesis; therefore he was transferred to the neurology ward. Head CT showed a hypodense area in the right thalamus corresponding to ischemic stroke. Four days later a follow-up MRI revealed areas of increased signal intensity on T2-weighted and FLAIR images involving almost the whole right thalamus with predominance of its anterior-medial portion and a small paramedian region within the left thalamus (Figure 3A). These regions showed
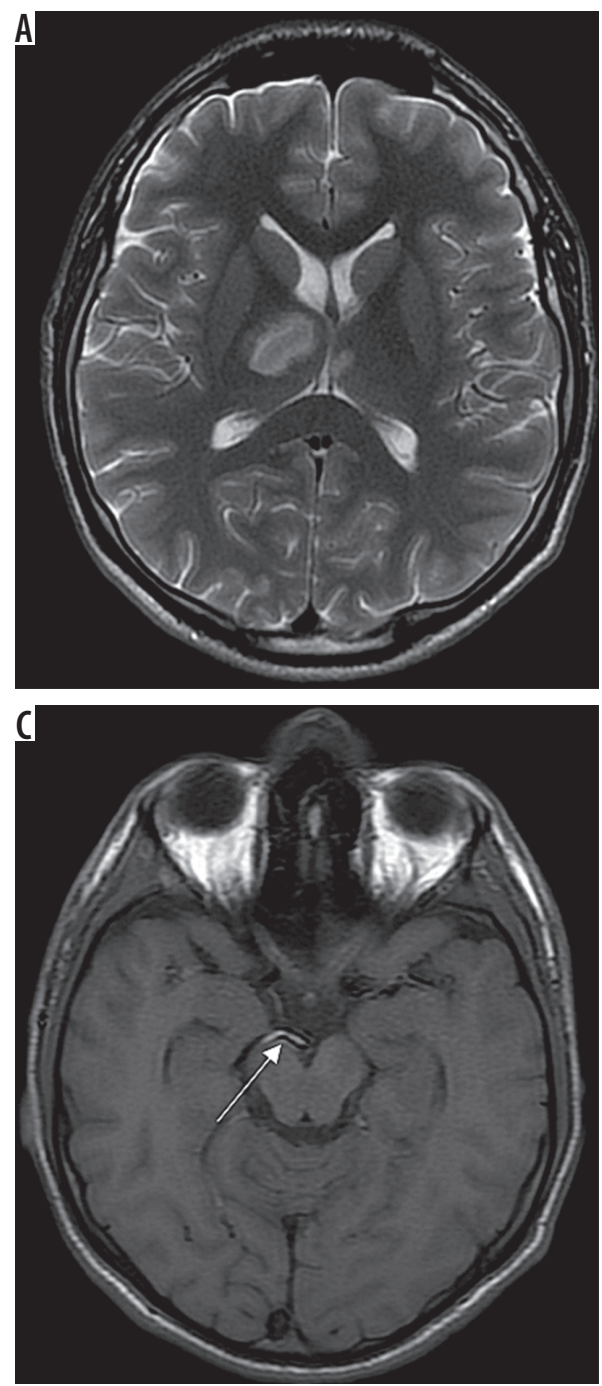

restricted diffusion on diffusion-weighted images (DWI) compatible with acute ischemia (Figure 3B).

The patient was treated neurologically for stroke. Due to anemia associated with recurrent bleeding, a total of $6 \mathrm{U}$ of packed red blood cells were transfused. The patient was discharged home 26 days later with improved cognitive and motor functions, with slight left-sided hemiparesis.

\section{Discussion}

Bleeding associated with post-tonsillectomy pseudoaneurysm occurs almost exclusively after 24 hours, usually 8 -30 days after surgery $[1,5,6]$. Recurrent post-tonsillectomy bleeding episodes with spontaneous cessation indicate the possibility of pseudoaneurysm formation and should be considered a warning sign of serious hemorrhage $[2,6]$. Facial and lingual arteries are most frequently involved due to their proximity to the inferior tonsillar pole, making this area especially vulnerable to injury if the sutures are placed deeply and hemostasis is achieved by electrocautery, which promotes necrosis $[3,5]$.
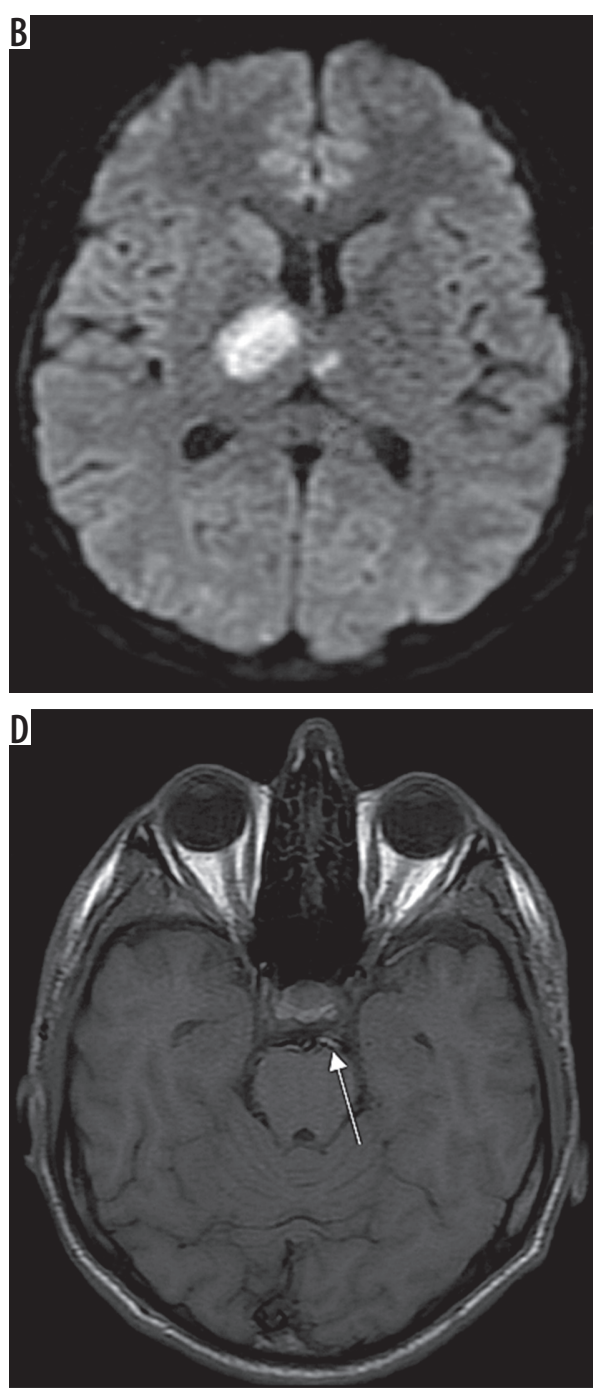

Figure 3. Magnetic resonance examination, axial T2-weighted (A), diffusion-weighted images (B) as well as T1-weighted images (C, D). The images show hyperintense regions of acute ischemic stroke involving predominantly the right thalamus as well as a small part of the left thalamus (A, B). On T1-weighted images $(C, D)$ the $P 1$ segments of both posterior cerebral arteries revealed high signal intensity more pronounced on the right side (arrows), probably due to P1 thrombus 
In the present case, the first 3 delayed bleeding episodes were severe but not life-threatening. No pulsating masses were observed in examination of the oral cavity, which, according to van Cruijsen et al., could be explained by the gradual development of pseudoaneurysm depending on the progressive dissolution of the clot and subsequent filling with blood of the periarterial space $[2,4]$. In addition, lying down in bed raises blood pressure in the carotid artery and increases the risk of bleeding from the aneurysm [4]. This occurred in our patient, who experienced a massive hemorrhage on the $20^{\text {th }}$ day and required immediate surgery.

Massive bleeding is life-threatening and requires immediate intervention and intensive care. Oral suture ligation is usually insufficient and ECA ligation is necessary. During the operation, the arteries should be checked for possible anastomoses. As reported by Schechter, such connections between the VA and the ECA or its branches are quite frequent and occur in as many as $1 \%$ of cerebral angiograms [7]. These collaterals are usually found between muscular branches of the VA and the occipital artery (OA), a branch of the ECA. They may be the cause of recurrent bleeding after ECA ligation and require exclusion of vascular anomaly. However, anastomoses may appear over time due to hemodynamic changes after surgery $[2,5,8,9]$. Due to the disturbance of normal pathways, the channels dilate to form alternate routes for the passage of blood to vital areas [2,7]. Flow direction (from VA to OA or from OA to VA) depends on hemodynamic conditions and on the mean pressure in each artery. Takeuchi et al. state that in patients who underwent ECA ligation, the main collateral pathways to the ECA were from the VA via occipital-vertebral anastomosis, making controlling bleeding not effective [8]. According to Dae-Chul, it is possible that the diastolic gradient may contribute to preferential flow from the VA to the OA since the diastolic flow component in the VA is higher than the equivalent in the ECA [10].

In our case the anastomosis was not ligated because it was not visible intraoperatively; however, it became significant over time due to the hemodynamic changes. This collateral channel gave rise to delayed recurrent bleeding from the tonsillar bed despite the prior ECA ligation. There was preferential flow regurgitation from the VA to the OA and the ECA above the ligation site, leading to hemorrhage and simultaneous steal phenomenon of the VA.

Severe post-tonsillectomy hemorrhage is treated with ECA ligation or, if it is non-life-threatening, with endovascular embolization of the injured artery, which seems to be more advantageous. Embolization has been reported as a safe and effective management in both post-tonsillectomy hemorrhage and pseudoaneurysms involving the ECA supply [1,2,4-6]. Diagnostic angiographic assessment and subsequent embolization can be accomplished during one procedure. Furthermore, the intra-arterial approach is less mutilating and is associated with a shorter time of hospitalization and convalescence. Feeding arteries can be indentified and selectively treated and potential anastomoses can be revealed. The possible procedure-related complications are perforation of the vessel or inadvertent embolism of the intact artery.

Among multiple embolic materials, coils are considered the preferred choice for post-tonsillectomy hemorrhage $[1,2,4]$. Coiling offers selective and permanent occlusion of the injured artery and is more controllable than polyvinyl alcohol (PVA) or liquid embolic agents, which bring a higher risk of reflux from the ECA into the internal carotid artery (ICA). In the present case, apart from using a coil, the authors additionally injected NBCA because there was no such risk since ECA ligation was performed above the bifurcation and they had vast experience in its use.

We believe that if the endovascular approach had been the initial treatment choice, it would have been possible to directly show the cause of the bleeding, which was the pseudoaneurysm, and to achieve target embolization, saving the ECA and protecting the patient from subsequent complications.

The patient developed bilateral thalamic infarct with onset of symptoms within the first 24 hours postoperatively. Thalamus is classically divided into 4 vascular territories: anterior, paramedian, inferolateral and posterior, supplied respectively by polar, paramedian, thalamogeniculate and posterior choroidal arteries [11]. However, one-third of patients manifest infarction outside the classical involvement, usually combining two or more of the typical locations. This refers to 3 variant distributions: anteromedian, central and posterolateral territory $[12,13]$. The most heterogeneous in its extent of vascularization is the paramedian artery, which in some cases can supply both the paramedian and anterior territories $[11,12]$. In most cases, the paramedian artery originates from the P1 segment of the posterior cerebral artery (PCA) on both sides. However, in one-fourth of cases there is a common trunk arising from one of the P1 segments providing bilateral distribution, called the artery of Percheron [11].

Bilateral thalamic infarcts are uncommon, with overall incidence of $0.6 \%$ in all cases of cerebral involvement [14]. The most common manifestation is paramedian artery territory infarction with either contralateral paramedian or thalamogeniculate artery territory involvement $[13,14]$. When there are bilateral paramedian thalamic infarctions, especially symmetrical, the presence of the Percheron artery should be suspected. The lack of visualization of this artery in magnetic resonance angiography (MRA), CTA or angiography does not exclude its presence because it can be occluded.

Our patient showed a bilateral manifestation of the thalamus infarct, but very asymmetric. The right thalamus was affected significantly with predominance in the anteriormedial portion, the left thalamus only in the small medial 
part. Such a manifestation, despite asymmetry, could correspond to the presence of the Percheron artery, although it could also result from occlusion of bilateral paramedian arteries arising from P1 segments of both PCAs as well. In MRI, T1-weighted images showed bilateral hyperintense P1 segments of PCAs and T2-weighted images also revealed hyperintense segments within both arteries, more extensive on the right side. These findings suggest bilateral thrombotic occlusion of $\mathrm{P} 1$ segments with predominance on the right side, which is correlated with larger ischemic involvement and excludes the initial hypothesis of the single Percheron artery.

Symptoms in our patient were typical of the anteromedian thalamic manifestation described in the literature [12-14] and included transient disturbances of consciousness, cognitive memory disorders and slight left-sided hemiparesis diminishing during hospitalization. However, the etiology of cerebral ischemic complication in the present case is unclear. The most frequent stroke mechanism for this territory is cardioembolism or artery-to-artery embolism [12-14]. The main risk factors are smoking, hypercholesterolemia, diabetes and hypertension. Our patient underwent echocardiography which showed no pathology. One-day delay of the onset of symptoms challenges the idea of iatrogenic stroke resulting from the migration of embolic material. Imaging examinations did not reveal the presence of NBCA depots in the thalamus. However, a thromboembolic mechanism seems to be probable taking into account the absence of comorbidities and MR findings. Perhaps coexisting anemia increased ischemia and had an impact on the state of consciousness. In the postoperative period, the patient was drowsy, which could prevent an accurate assessment of his neurological condition and mask the symptoms of stroke.

In conclusion, this case report shows the importance of an anastomosis between the ECA and the vertebrobasilar system, both for recurrence of significant post-tonsillectomy bleeding and for potential thromboembolic complications. It is therefore very important, whenever ECA ligation is considered, to exclude aberrant arterial blood supply by collateral pathways outgoing from the ECA. Whenever episodes of gushing secondary bleeding with spontaneous cessation occur, the presence of pseudoaneurysm should be suspected.

The present case is complex and the etiology of neurological complications remains unclear, but we believe that they are procedure-related and not NBCA-related. In our opinion, embolization is a better therapeutic option in post-tonsillectomy hemorrhage and should be performed as selectively as possible. Nevertheless, liquid embolic materials should be avoided.

\section{Conflict of interest}

The authors report no conflict of interest.

\section{References}

1. Opatowsky MJ, Dale Browne J, McGuirt Jr WF, et al. Endovascular treatment of hemorrhage after tonsillectomy in children. Am J Neuroradiol 2001; 22: 713-716

2. Cohen JE, Gomori JM, Moscovici S, et al. Endovascular management of postoperative pseudoaneurysms of the external carotid artery. J Clin Neurosci 2012; 19: 649-654.

3. Windfuhr JP, Verspohl BC, Chen YS, et al. Post-tonsillectomy hemorrhage - some facts will never change. Eur Arch Otorhinolaryngol 2015; 272: 1211-1218.

4. van Cruijsen N, Gravendeel J, Dikkers FG. Severe delayed posttonsillectomy haemorrhage due to a pseudoaneurysm of the lingual artery. Eur Arch Otorhinolaryngol 2008; 265: 115-117.

5. Choi KJ, Cheng T, Cobb MI, et al. Recurrent post-tonsillectomy bleeding due to an iatrogenic facial artery pseudoaneurysm. Acta Otolaryngol Case Rep 2017; 2: 103-106.

6. Windfuhr JP, Sesterhenn AM, Schloendorff G, et al. Post-tonsillectomy pseudoaneurysm: an underestimated entity? J Laryngol Otol 2010; 124: 59-56.

7. Schechter MM. The occipital-vertebral anastomosis. J Neurosurg 1964; 21: 758-762.

8. Takeuchi Y, Suzuki H, Numata T, et al. Hemodynamic changes in the head and neck after ligation of the unilateral carotid arteries: a study using color Doppler imaging. Ann Otol Rhinol Laryngol 1994; 103: 41-45.

9. Harada J, Kuwayama N, Nishijima M, et al. Symptomatic occipitalvertebral anastomosis: a case report. Interv Neuroradiol 2000; 6: 317-320.

10. Harada J, Kuwayama N, Nishijima M, et al. Symptomatic occipitalvertebral anastomosis: a case report. Interv Neuroradiol 2000; 6: 317-320.

11. Percheron G. The anatomy of the arterial supply of the human thalamus and its use for the interpretation of the thalamic vascular pathology. Z Neurol 1973; 205: 1-13.

12. Li S, Kumar Y, Gupta N, et al. Clinical and neuroimaging findings in thalamic territory infarctions: a review. J Neuroimaging 2018; 28: 343-349.

13. Kumral E, Deveci EE, Colak AY, et al. Multiple variant type thalamic infarcts: pure and combined types. Acta Neurologica Scandinavica 2015; 131: 102-110.

14. Kumral E, Evyapan D, Balkır K, et al. Bilateral thalamic infarction: clinical, etiological and MRI correlates. Acta Neurologica Scandinavica 2001; 103: 35-42. 